Supporting Information for:

A Closer Look into the Traditional Purification Process of CdSe Semiconductor Quantum Dots

Behtash Shakeri and Robert W. Meulenberg

University of Maine, Orono ME
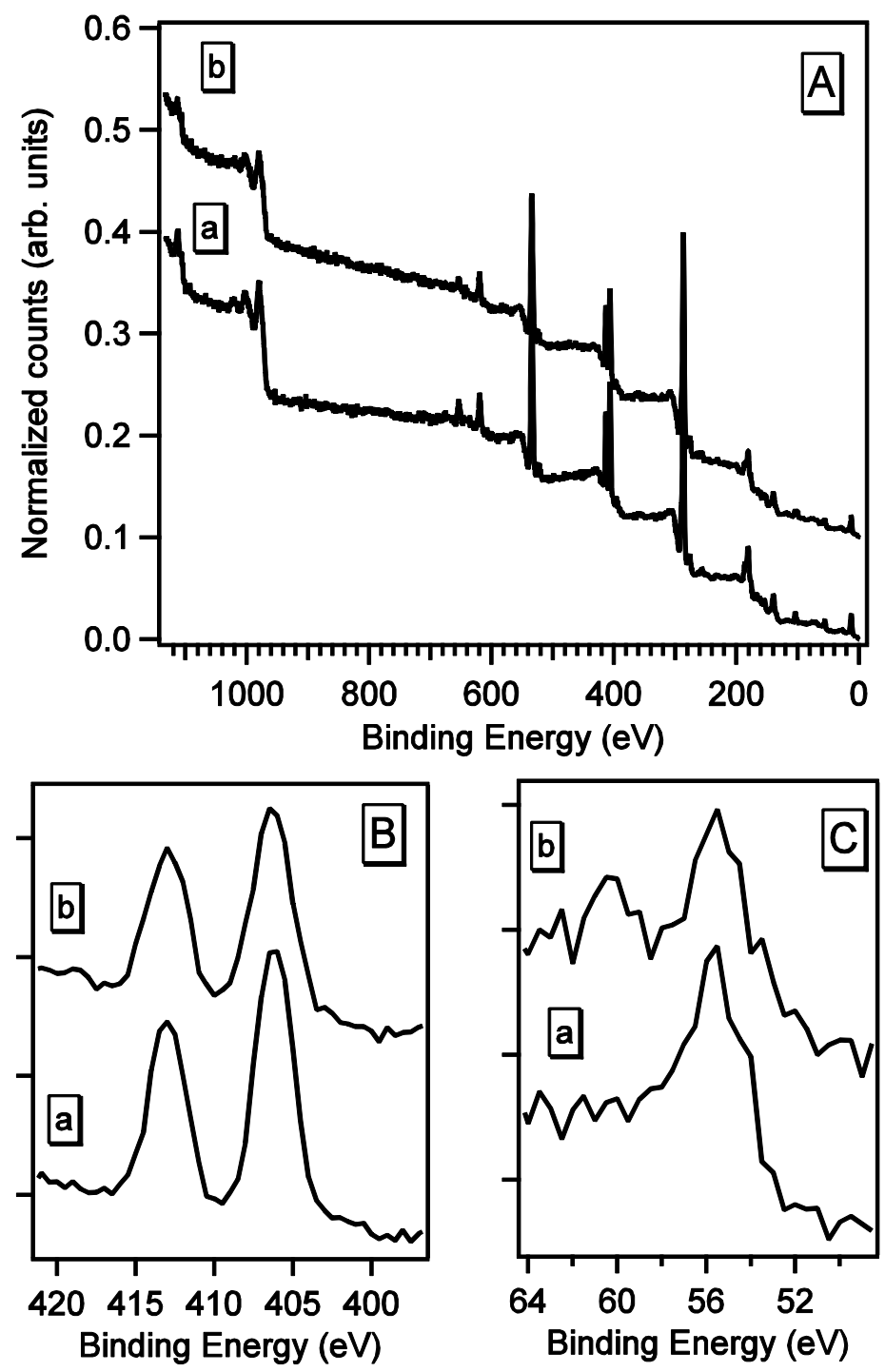

Figure S1. (A) Survey, (B) Cd $4 p$, and (C) Se 2p x-ray photoelectron spectra for (a) zero and (b) eight washes for $4.8 \mathrm{~nm}$ CdSe QDs 


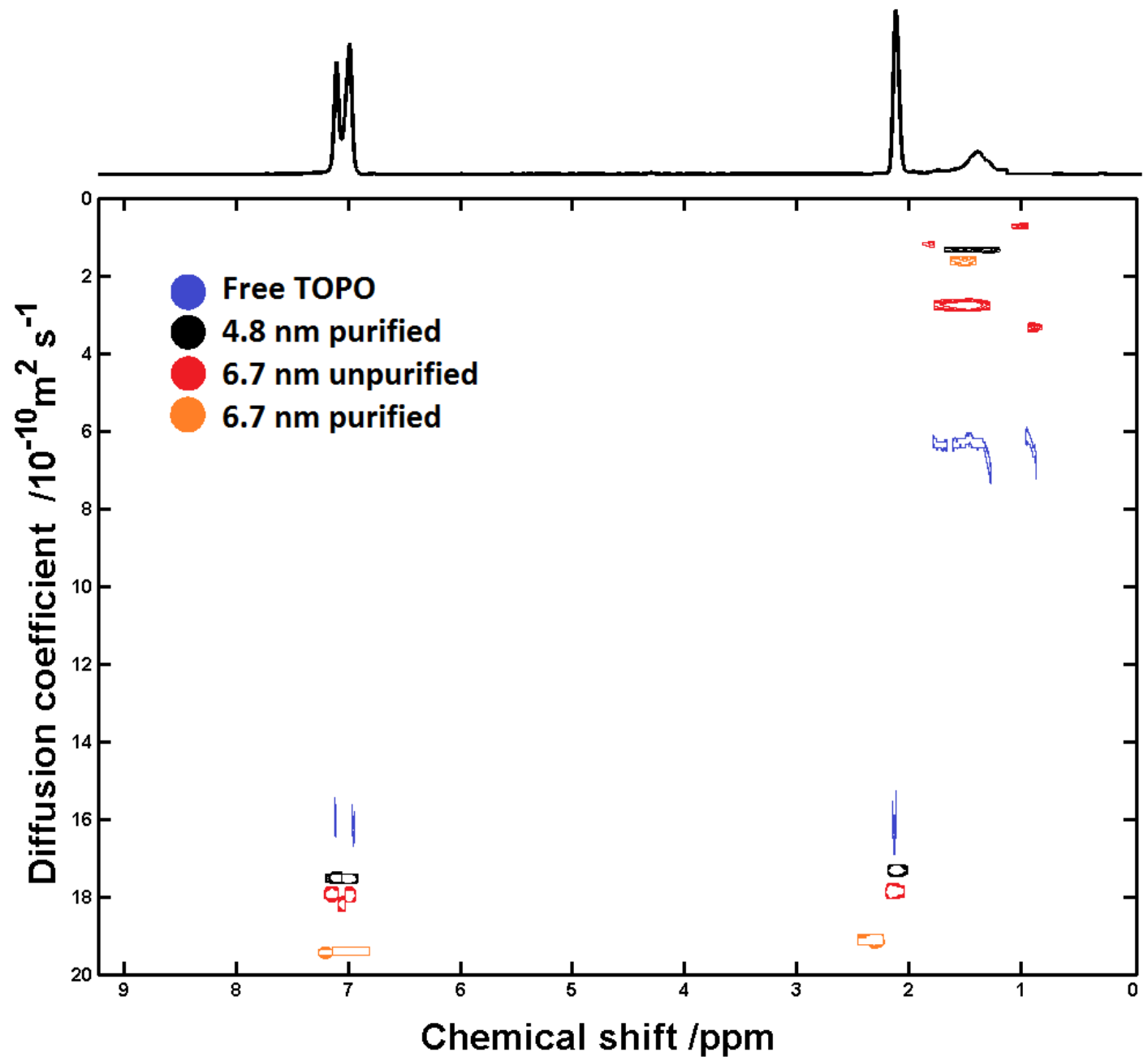

Figure S2. ${ }^{1} \mathrm{H}$ DOSY spectra of unpurified and purified CdSe QDs compared to the free ligand TOPO. 


\section{Electrostatic Model}

Consider a "fully passivated" QD; this corresponds to a sample that has been washed \$sim\$ one time. In this case, there are no free ligands and the QD contains both L- and X-types ligands. At this point, the overall charge of the QD sample is negative, as the X-type ligands ensure charge neutrality despite the materials being Cd-rich. After 3-4 washes, the QDs no longer have L-type (or neutral) ligands and then the X-type ligands will be removed in the next processing steps. If one considers that for every X-type ligand removed the QD gains +1 in charge, then we can calculate the electrostatic energy as ligands are sequentially removed. We can begin with the Coulomb energy between a removed ligand and the QD,

$$
E=\frac{q_{L} q_{Q D}}{4 \pi \epsilon \epsilon_{o} R}
$$

where $q_{L}$ and $q_{Q D}$ are the charge of the ligand and QD, respectively, and $\varepsilon$ and $\varepsilon_{0}$ are the permittivity and dielectric constant, respectively. As we remove ligands, the QD builds up charge and the Coulomb energy can simply be expressed as,

$$
E=\frac{e^{2}}{4 \pi \epsilon \epsilon_{o} R} \sum_{n=1}^{n_{f}} n
$$

where $n$ is an integer that counts the numbers of ligands removed (or the built up charge on the QD). Figure S3 plots the Coulomb energy as a function of QD charge as the distance that the ligand is from the QD surface is varied. We assume that the energy needed to remove a chemisorbed ligand from the surface is supplied through the system (i.e. QD phonon, ligand vibrations, solvation, etc.). As the ligand leaves the particle surface, the Coulomb interaction between the outgoing ligand and the QD will then have to be considered. For one ligand removed, the QD charge is +1 and the Coulomb energy is very small (10s to $100 \mathrm{~s}$ of meV) and ligand removal is easy. As more ligands are removed, the Coulomb interaction between the outgoing ligand and QD increases, and ligand removal becomes more difficult. For instance, consider the case where 10 ligands are removed. For this case, the Coulomb energy varies as high as $10 \mathrm{~s}$ of $\mathrm{eV}$, and in the case for 20 ligands removed, the Coulomb energy is even greater. 


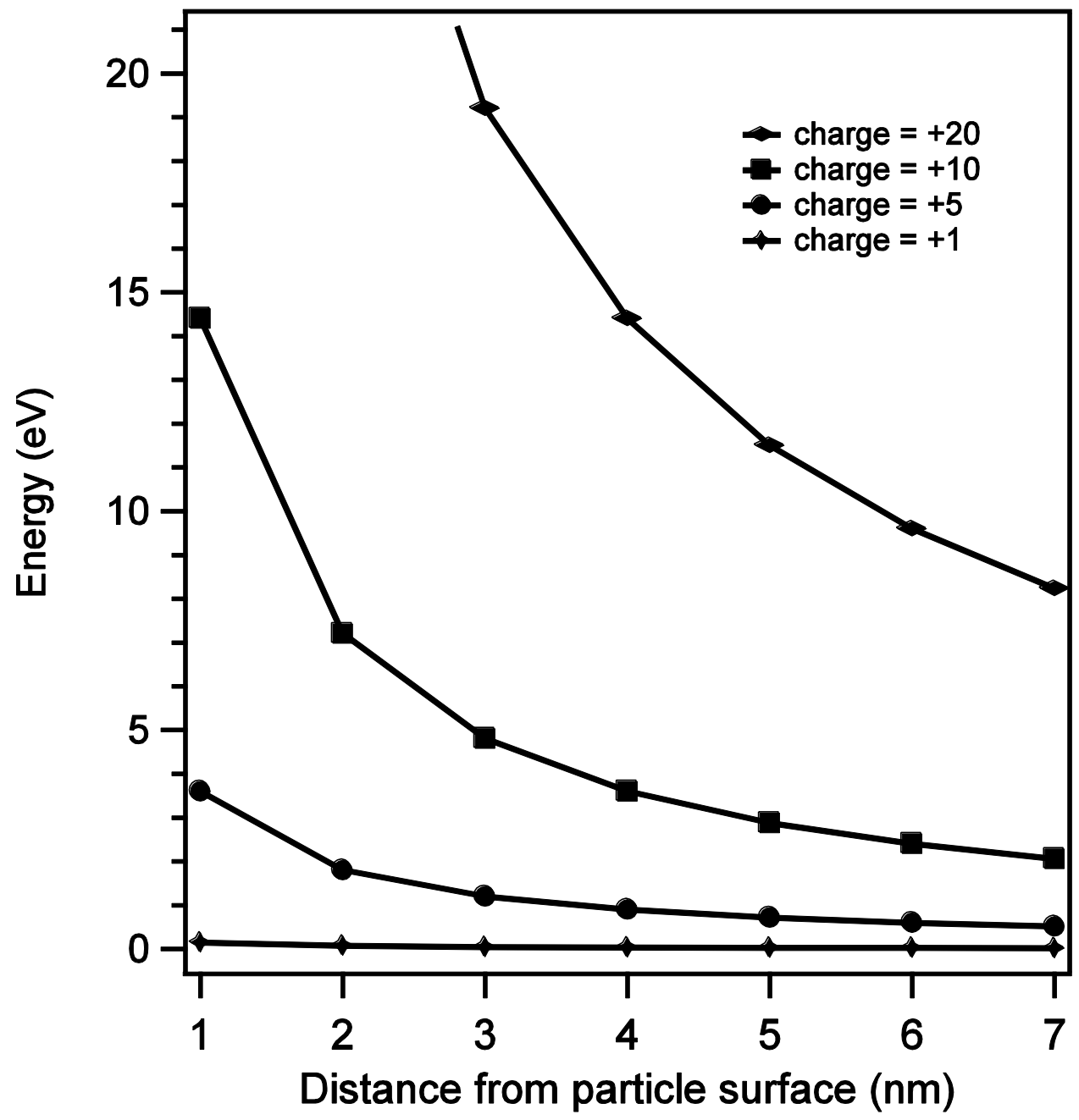

Figure S3. Electrostatic energy between a single -1 charge ligand and a QD with varying charge, plotted as a function of the distance the ligand is form the QD surface. 